\title{
Artificial Rupture of Membrane
}

National Cancer Institute

\section{Source}

National Cancer Institute. Artificial Rupture of Membrane. NCI Thesaurus. Code C92734.

Perforation of fetal membranes to facilitate childbirth. 